\title{
Correspondence
}

\section{Cystic fibrosis and myocardial fibrosis}

Sir,

We were interested in the case reported by Mukherji et al. (1976), which suggested that cystic fibrosis might be an acid mucopolysaccharidosis. We have seen a similar child. A male was referred to hospital elsewhere aged 20 months with history of diarrhoea from birth and poor appetite. On examination, abdomen distended, height and weight on the 3rd centile. Faecal fat excretion 11.9 $\mathrm{mmol} / 24 \mathrm{~h}(3.3 \mathrm{~g} / 24 \mathrm{~h})$. Impaired xylose absorption test. Sweat sodium $34 \mathrm{mmol} / 1$ (34 $\mathrm{mEq} / \mathrm{l})$. A presumptive diagnosis of coeliac disease was made but an intestinal biopsy was not carried out. A gluten-free diet was introduced. On the diet his temperament was thought to have improved as had the tendency to diarrhoea. He had had no serious respiratory tract infections up to that time.

He was seen in Swindon aged $3 \frac{1}{2}$ years. Height and weight remained below the 3 rd centile. He was pale, haemoglobin $8.6 \mathrm{~g} / \mathrm{dl}$. The film showed marked hypochromia. Anaemia responded rapidly to iron by mouth. He was passing frequent pale stools, but the gluten restriction was not being maintained strictly. Parental permission to carry out intestinal biopsy was initially withheld, but their anxieties were eventually overcome and biopsy was performed aged $4 \frac{1}{2}$ years. A partial villous atrophy was found. 5-day fat balance confirmed a steatorrhoea with fat excretion of $38.7 \mathrm{mmol} / 24 \mathrm{~h}$ (11 g /24 h); normal $<14 \cdot 1 \mathrm{mmol} / 24 \mathrm{~h}(<4 \mathrm{~g})$. Faecal tryptic activity was detected to a dilution of 1 in 80 , but sweat chloride levels were $80 \mathrm{mmol} / 1$ ( $80 \mathrm{mEq} / \mathrm{l})$ on consecutive samples. A diagnosis of cystic fibrosis was made, possibly associated with gluten intolerance as previously described (Hide and Burman, 1969). At the same time a younger brother was diagnosed as having cystic fibrosis. He also had some villous changes on jejunal biopsy. Pancreatic supplements were added to gluten-free regimens.

Aged 5 years he was admitted to hospital with pneumonia. In spite of conventional treatment with cloxacillin and colistin he collapsed and died $\mathbf{4 8}$ hours after admission. At necropsy (Dr. A. K. Davis) trachea, bronchi. and bronchioles contained viscid green sputum from which Staphylococcus aureus was cultured. The lungs showed gross pneumonic consolidation. The heart was not enlarged, but the myocardium showed a grossly abnormal appearance with variations in thickness and extensive areas of firm pale tissue. These changes were most marked in the anterior wall of the left ventricle, but were present throughout the myocardium. The pericardium, endocardium, and coronary arteries were normal.

Histology of the heart muscle showed very dramatic changes with severe replacement fibrosis distributed patchily through the myocardium. Large tracts of muscle were wholly or largely replaced by fairly cellular fibrous tissue. Histochemical staining methods for mucopolysaccharides were applied (Periodic Acid Schiff and Alcian Blue), which showed a mixture of neutral and acid mucopolysaccharides in the heart muscle, both in the fibrotic areas and between the muscle cells. This similar mixture of neutral and acid mucopolysaccharides was shown in both the pancreas and the salivary glands.

There have now been a number of reports of myocardial fibrosis complicating cystic fibrosis (Barnes et al., 1970; Oppenheimer and Esterly, 1973). In the majority of these histology of the myocardium showed replacement of muscle fibres by simple fibrous connective tissue and where special stains to show mucopolysaccharides were carried out, these proved negative. The case reported by Mukherji et al. and this case are the first in which mucopolysaccharide deposits have been found in the myocardium. The mixture of neutral and acid mucopolysaccharides in our case does not support the hypothesis that cystic fibrosis is simply a genetic acid mucopolysaccharidosis. The reports of heart muscle disease and cystic fibrosis suggest the value of reviewing the electrocardiogram from time to time in the follow-up of children with cystic fibrosis.

\section{DAVID W. HIDE Department of Paediatrics, St. Mary's Hospital, Newport, Isle of Wight. \\ ROBERT MARTLEW Department of Pathology, Princess Margaret Hospital,} Swindon.

\section{References}

Barnes, G. L., Gwynne, J. F., and Watt, J. M. (1970). Myocardial fibrosis in cystic fibrosis of the pancreas. Australian Paediatric Journal, 6, 81-87.

Hide, D. W., and Burman, D. (1969). An infant with both cystic fibrosis and coeliac disease. Archives of Disease in Childhood, 44, 533-535.

Mukherji, R. N., Moss, P. D., and Heffernan, D. K. (1976). Is cystic fibrosis an acid mucopolysaccharidosis? Archives of Disease in Childhood, 51, 563-564.

Oppenheimer, E. H., and Esterly, J. R. (1973). Myocardial lesions in patients with cystic fibrosis of the pancreas. Johns Hopkins Medical Journal, 133, 252-261. 